\title{
EFEITOS DA ESCARIFICAÇÃO NA QUALIDADE FÍSICA DE UM LATOSSOLO VERMELHO DISTROFÉRRICO APÓS TREZE ANOS DE SEMEADURA DIRETA(1)
}

\author{
M. A. ARAUJ $0^{(2)}$, C. A. TORMENA(3), T. T. INOUE(4) \& A. C. S. COSTA(3)
}

\begin{abstract}
RESUMO
O objetivo deste trabal ho foi avaliar as alterações na qualidade física de um Latossolo Vermelho distroférrico provocadas pela escarificação, após 13 anos de semeadura direta. O experimento constituiu-se de dois tratamentos: escarificação do solo a 0,30 m de profundidade (SDE) e testemunha mantendo a semeadura direta (SD). Amostras de solo, com estrutura não deformada, foram coletadas nas profundidades de 0-0,15 $\mathrm{m}$ e 0,15-0,30 m, a partir das quais foram determinados a curva de retenção de água, a curva de resistência do solo à penetração, a densidade do solo e o "Intervalo Ótimo de Tensão da Água no Solo" (IOP). Os resultados mostraram que, na profundidade de 0-0,15 m, a escarificação promoveu modificações na porosi dade do solo, manten do condições adequadas de aeração em tensões matriciais menores do que 0,01 MPa. Por outro lado, no solo escarificado, constatou-se a maior ocorrência de limitações pela resistência do solo à penetração em tensões menores do que 1,5 MPa. Para a camada de 0,15-0,30 m, no tratamento SDE, a resistência do solo à penetração foi o limite superior do IOP. Os resultados deste estudo sugerem que, neste solo e sob esta condição de manejo, não é necessária a escarificação.
\end{abstract}

Termos para indexação: resistência à penetração, semeadura direta, estrutura do solo, intervalo ótimo de tensão da água no solo (IOP).

(1) Trabalho desenvolvido no Laboratório de Física de Solos do Curso de Agronomia da Universidade Estadual de Maringá - UEM. Recebido para publicação em dezembro de 2003 e aprovado em maio de 2004.

(2) Doutorando do Programa de Pós-Graduação em Agronomia da Universidade Estadual de Maringá - UEM. Av. Colombo 5790, CEP 87020-900 Maringá (PR). Bolsista da CAPES. E-mail: araujomaa@yahoo.com.br

(3) Professor do Departamento de Agronomia, UEM. Bolsista do CNPq. E-mail: catormena@uem.br; acscosta@uem.br

(4) Professor do Curso de Agronomia do Integrado, Colégio e Faculdade. Av. Irmãos Pereira 670, Centro, CEP 87301-010 Campo Mourão (PR). E-mail: ttinoue@yahoo.com.br 


\title{
SUMMARY: EFFECTS OF CHISELING ON PHYSICAL QUALITY OF A DYSTROFERRIC RED LATOSOL AFTER THIRTEEN YEARS OF NO-TILLAGE
}

\begin{abstract}
The objective of this study was to evaluate alterations in the physical soil quality of a Dystroferric Red Latosol (Typic Hapl orthox) caused by chiseling, after thirteen years of notillage. Theexperiment consisted of two management systems: soil chiseling down toa $0.30 \mathrm{~m}$ depth (NTC) and no-tillage (NT). Undisturbed soil samples were collected at depths of 0$0.15 \mathrm{~m}$ and $0.15-0.30 \mathrm{~m}$, and thesoil water retention curve, thesoil resistanceto penetration curve, the soil bulk density, and the "Least Limiting Water Potential" (LLWP) were determined. The results showed that the chiseling caused modifications in soil porosity in the $0-0.15 \mathrm{~m}$ layer, maintaining appropriateaeration conditions in water matric potentials under $0.01 \mathrm{MPa}$. On theother hand, thestrongest limitations by soil penetration resistance in matric potentials under $1.5 \mathrm{MPa}$ wereverified in theNTC soil management. In the 0.15$0.30 \mathrm{~m}$ soil layer under the NTC treatment, the soil penetration resistance was the upper limit of the LLWP. The results of the study indicate that under the present management condition, chiseling is not necessary in this soil.
\end{abstract}

Indexterms: soil penetration resistance, no-tillage, soil structure, least limiting water potential (LLWP).

\section{INTRODUÇÃO}

Os sistemas de uso e manejo podem influir nas propriedades físicas do solo e na produtividade das culturas. Nos solos das regiões tropicais e subtropicais, a adoção de sistemas de manejo com mínimo revolvimento é fundamental para a conservação do sol o e da água e acúmulo de matéria orgânica, com vistas em garantir elevadas produtividades com reduzido impacto ambiental. Neste contexto, o sistema de semeadura direta ocupa cerca de 18.000.000 ha e está em plena expansão no Brasil (FEDERAÇÃO BRASILEIRA DE PLANTIO DIRETO NA PALHA, 2004). No entanto, apesar das inúmeras vantagens da semeadura direta, temse verificado que, em sol os argil osos, a compactação superficial do solo pode impor limitações à produtividade das culturas.

A compactação superficial na semeadura direta depende, princi palmente, do reduzido revol vimento do solo, da acomodação natural de partículas e do tráfego contínuo de máquinas e implementos na superfície do solo (Mahboubi et al., 1993; Stone \& Silveira, 2001). Isto resulta em aumento da densidade do sol o e modificações no sistema poroso (Hill, 1990; De Maria et al., 1999; Beutler et al., 2001), bem como em aumento na resistência do solo à penetração (Beutler et al., 2001; Costa et al., 2003). Estas modificações podem ter impactos negativos no crescimento das raízes e da parte aér ea das plantas (Kirkegaard et al., 1995).

O controle da compactação superficial do solo submetido à semeadura direta pode ser feito por métodos culturais (Müller et al., 2001) e mecânicos (Hamilton-Manns et al., 2002) e não há estudos conclusivos a respeito deste assunto. Várias pesquisas demonstram que a escarificação do solo promove a redução da densidade e da resistência do solo à penetração (De Maria et al., 1999), com o mínimo possível de movimentação do solo. A longevidade dos efeitos da escarificação é muito variável, desde poucos meses (Evans et al., 1996; Hamilton-Manns et al., 2002) até alguns anos (Twonlow et al., 1994).

Conforme Torres et al. (1998), a escarificação preserva grande parte dos resíduos na superfície, com pequenoimpacto nos teores de matéria orgânica do solo. Neste sentido, Torres \& Saraiva (1998) indicaram que a melhoria das condições físicas do solo tem reflexos positivos na produtividade da cultura da soja. Apesar dos efeitos positivos da escarificação, existem evidências de que estes efeitos são de curta duração. Por exemplo, Busscher et al. $(1995,2002)$ constataram que o efeito da subsol agem é temporário, uma vez que a reconsolidação do solo aumenta com o vol ume cumulativo de preci pitações. Segundo esses autores, em solos estruturados, a reconsolidação pode ser influenciada pela dinâmica da água no espaço poroso inter e intra-agregados e pode ser afetada também pel o selamento superficial ou pela estabilidade estrutural que pode modificar a quantidade de água que infiltra no solo.

O carbono orgânico (CO) influencia o comportamento físico do sol o e, segundo Kay \& Angers (1999), a forma e a estabilidade da estrutura do solo geralmente melhoram com o aumento do conteúdo de matéria orgânica do solo. A mobilização do solo por equipamentos de haste e, ou, discos também pode promover redução no teor deCO do sol o (Mahboubi et al., 1993), com reflexos nas suas propriedades físicas. 
Bayer et al. (2003) encontraram maior teor de CO no solo cultivado no sistema de semeadura direta em comparação ao preparo convencional e preparo reduzido e atribuíram estes resultados ao ambiente menos oxidativo na semeadura direta. Práticas de manejo que contribuem para a aceleração do processo de oxidação da matéria orgânica favorecem o aumento na taxa de dispersão de argila, a qual, segundo Kay \& Angers (1999), pode resultar em aumento na resistência do solo pelo fato de a argila dispersa funcionar como material cimentante com o secamento do solo. Além disso, de acordo com Mahboubi \& Lal (1998), orevolvimento do solo, além de provocar perdas na sua qual idade estrutural, afeta a atividade da fauna do solo, especialmente das minhocas.

Diferentes propriedades físicas do solo têm sido empregadas para quantificar as mudanças causadas pelo manejo do solo (Karlen \& Stott, 1994). Em geral, são utilizadas propriedades físicas individualmente, não levando em conta as possíveis interações entre elas. De acordo com Letey (1985), os atributos físicos do solo com influência direta no desenvolvimento das plantas são: temperatura, retenção de água pelo solo, aeração e resistência do soloà penetração. Vários trabalhos demonstram que há uma interdependência entre estas propriedades. Por exemplo, uma variação na umidade do solo influencia a taxa de difusão de oxigênio no solo, a resistência do solo à penetração, a tensão e a disponibilidade de água no solo às plantas, entre outras propriedades. Neste sentido, a resistência do solo à penetração varia inversamente com a umidade (Silva et al., 1994; Tormena et al., 1998) e positivamente com a densidade do sol o (Tormena et al., 1999a,b; I mhoff et al., 2001). De acordo com Granatstein \& Bezdicek (1992), a interação dessas propriedades físicas determina a qualidade do solo.

O conceito do "Least Limiting Water Range" (LLWR) foi quantificado por Silva et al. (1994) a partir da proposta de Letey (1985). No Brasil, o LLWR foi quantificado por Tormena et al. (1998), que o traduziu como I ntervalo Hídrico Ótimo (I HO), sendo definido como a faixa de água do solo em que são mínimas as limitações ao desenvolvimento de plantas associadas à disponibilidade de água, aeração e resistência do solo à penetração. O I HO tem sido utilizado como um índice da qual idadefísica e estrutural do solo (Silva et al., 1994; Sharma \& Bhushan, 2001; Wu et al., 2003). Kay (1990) estabeleceu que quanto menor oval or do l $\mathrm{HO}$ maior será a probabilidade de ocorrência de estresses físicos por reduzida aeração ou excessiva resistência do solo à penetração, acarretando reduzida qualidade física do solo para o desenvol vimento das culturas.

Vários trabalhos têm indicado que o IHO pode também ser expresso em termos de tensão em vez do conteúdo deágua do solo (Boone et al., 1986, 1987;
Boone, 1988; Boone \& Veen, 1994; Hakansson \& Lipiec, 2000; Lipiec \& Hakansson, 2000). Desta forma, o termo IHO pode ser expresso como "I nterval o Ótimo de Tensão da Água no Solo" (IOP), com o mesmo significado e definição do IHO (Tormena et al., 1999a). De acordo com J ensen et al. (1998), a tensão da água no solo está mais intimamente relacionada com o crescimento das plantas do que o conteúdo de água disponível.

O objetivo destetrabal ho foi quantificar os efeitos da escarificação na qualidade física e estrutural de um Latossolo Vermel ho distroférrico cultivado por 13 anos no sistema de semeadura direta.

\section{MATERIAL E MÉTODOS}

O experimento foi realizado no município de Campo Mourão, PR, situado a $24^{\circ} 02^{\prime} 38^{\prime \prime}$ Sul e $52^{\circ}$ $22^{\prime} 40^{\prime \prime}$ Oeste, apresentando al titude média de $630 \mathrm{~m}$ e com médias anuais de temperatura e preci pitação de $20^{\circ} \mathrm{C}$ e $1.340 \mathrm{~mm}$, respectivamente. Segundo a classificação de Köppen, o clima é do tipo Cfa (subtropical úmido mesotérmico). No local do experimento, o sol o foi classificado como Latossolo Vermel ho distroférrico (E mbrapa, 1999), a análise granulométrica realizada na camada de $0-0,20$ m de profundidade indicou valores médios de $660 \mathrm{~g} \mathrm{~kg}^{-1}$ de argila, $240 \mathrm{~g} \mathrm{~kg}^{-1}$ de silte e $100 \mathrm{~g} \mathrm{~kg}^{-1}$ de areia, classe textural muito argilosa (E mbrapa, 1999).

O experimento foi realizado em uma propriedade rural com fins comerciais, que vinha sendo submetida ao sistema de semeadura direta por 13 anos consecutivos, utilizando a rotação milho/ aveia/soja/trigo/soja/trigo. Foram demarcadas duas áreas contíguas, com $2500 \mathrm{~m}^{2}$ cada uma. Numa dessas áreas, a escarificação foi feita em abril de 2001, na profundidade de 0-0,30 m, antes da semeadura do trigo, utilizando-se um escarificador da marca ikeda com quatro hastes e munido de um rolo destorroador. Desta forma, os tratamentos constituíram-se de semeadura direta (SD) e semeadura direta + escarificação (SDE). O solo apresentava umidade bem abaixo da capacidade de campo quando da realização da escarificação.

A amostragem foi realizada seis meses após a escarificação do solo (outubro de 2001), em seqüência à colheita do trigo e antes da semeadura da soja. Foram selecionados, al eatoriamente, 28 pontos de amostragem em cada tratamento. Em cada ponto, no centro da camada de 0-0,15 m e 0,15-0,30 m, respectivamente, foram coletadas amostras com estrutura não deformada em anéis metálicos $(0,05 \mathrm{~m}$ de diâmetroe 0,05 m dealtura), perfazendo um total de 112 amostras. Em seguida, as amostras foram envoltas em papel alumínio, acondicionadas em sacos plásticos e mantidas sob temperatura de aproximadamente $5^{\circ} \mathrm{C}$ até serem analisadas. 
Também foram coletadas amostras deformadas na profundidade de 0-0,15 m para fins da determinação do CO do solo, utilizando o método descrito por Embrapa (1997).

Após devidamente preparadas, as amostras com estrutura não deformada foram saturadas por meio de el evação gradual de uma lâmina deágua em uma bandeja. Para determinar a curva de retenção, foi adotado o procedimento descrito em Silva et al. (1994), as amostras foram divididas em sete grupos de 16 amostras, sendo quatro amostras por tratamento e profundidade de amostragem em cada tensão utilizada. Foram utilizadas as seguintes tensões: 0,006, e 0,01 MPa numa mesa de tensão adaptada de Kiehl (1979) e, 0,033, 0,07, 0,1, 0,45 e 1,5 M Pa, utilizando pressões aplicadas em câmaras com placas porosas (K lute, 1986).

Atingindo o equilíbrio hídrico em cada tensão, as amostras foram pesadase, em seguida, determi nada a resistência do solo à penetração, utilizando o penetrômetro el etrônico e o método descrito por Tormena et al. (1998). Após a determinação da resistência, as amostras foram secas em estufa a $\pm 105^{\circ} \mathrm{C}$, por $24 \mathrm{~h}$, para a determinação da umidade e da densidade do solo (Blake \& Hartge, 1986).

Para avaliar o IOP, énecessário ajustar aos dados experimentais model os matemáticos que descrevam as curvas de retenção de água e de resistência do sol o. A curva de retenção de água (CRA) é utilizada para estimar o tensão da água no solo na qual o volume de poros com ar atinge $10 \%$ ou $0,1 \mathrm{~m}^{3} \mathrm{~m}^{-3}$. Para o ajuste da curva de retenção de água, empregou-se a função utilizada por Ross et al . (1991), descrita na equação 1:

$$
\tau=\mathrm{a} \theta^{\mathrm{b}}
$$

que, com a aplicação da função logarítmica, resulta numa função linear descrita pela equação 2:

$$
\ln (\tau)=\ln (a)+b \ln (\theta)
$$

em que $\theta=$ conteúdo vol umétrico de água $\left(\mathrm{m}^{3} \mathrm{~m}^{-3}\right)$, $\tau=$ tensão matricial (MPa), e a e b são coeficientes obtidos no ajuste do model o. Os efeitos da densidade do solo, dos tratamentos e das profundidades estudados sobrea CRA (variáveis qualitativas: SDE $=0, \mathrm{SD}=1$; profundidade $0-0,15 \mathrm{~m}=0 \mathrm{e}$ profundidade $0,15-0,30 \mathrm{~m}=1$ ) foram avaliados conforme Silva \& Kay (1997).

A resistência do sol o à penetração (RP) varia com o conteúdo deágua $(\theta)$, que pode ser substituído pela tensão matricial $(\tau)$, e com a densidade do solo (Ds), de modo que, a partir desta relação funcional entre RP, Ds e $\tau$, pode-se determi nar a curva de resistência do solo (CRS). A CRS foi ajustada por um modelo não-linear proposto por Busscher (1990), utilizando a tensão matricial $(\tau)$, em vez da umidade $(\theta)$ como variável independente do modelo. O modelo utilizado é descrito na equação 3.

$$
R P=c \tau^{d} D s^{e}
$$

que, com a transformação logarítmica, resulta na equação 4:

$$
\ln (R P)=\ln (c)+d \ln (\tau)+e \ln (D s)
$$

sendo RP = resistência do solo à penetração ( $\mathrm{MPa})$, $\tau=$ tensão $(\mathrm{MPa}), \mathrm{Ds}=$ densidade do solo $\left(\mathrm{Mg} \mathrm{m}^{-3}\right)$, e $c$, d, e e os coeficientes do modelo. O procedimento de ajuste da função dos dados ao model o foi similar ao adotado para a curva de retenção deágua no sol o. A influência das variáveis qualitativas (variáveis qualitativas: $\mathrm{SDE}=0, \mathrm{SD}=1$; profundidade $0-0,15 \mathrm{~m}$ $=0$ e profundidade $0,15-0,30 \mathrm{~m}=1$ ) foi avaliada conforme Silva \& Kay (1997).

O IOP foi determinado adotando os valores críticos associados com $\tau$, resistência do solo à penetração e porosidade de aeração, que foram, respectivamente: a capacidade de campo $\left(\tau_{\mathrm{CC}}\right)$ ou a $\tau=0,01 \mathrm{MPa}$ (Reichardt, 1988); o ponto de murchamento permanente ( $\left.\tau_{\text {PMP }}\right)$ ou a $\tau=1,5 \mathrm{MPa}$ (Savage et al., 1996); a RP $=2,0 \mathrm{MPa}$ (Taylor et al., 1966) e a porosidade de aeração de $0,10 \mathrm{~m}^{3} \mathrm{~m}^{-3}$ (Grable \& Siemer, 1968). Os valores da $\tau$ nos quais a $\mathrm{RP}=2,0 \mathrm{MPa}$ foram obtidos por meio da curva de resistência do solo ajustada aos dados. O conteúdo de água em que a porosidade de aeração é de $0,10 \mathrm{~m}^{3} \mathrm{~m}^{-3}$ foi obtido pela subtração de $0,10 \mathrm{~m}^{3} \mathrm{~m}^{-3}$ do val or da porosidadetotal. Esteconteúdo de água foi utilizado para se obter o respectivo valor de $\tau$, utilizando a curva de retenção de água no solo. O IOP foi calculado como a diferença entre o limite superior e inferior dos valores de $\tau$. O limite inferior foi considerado o mai or val or de $\tau$, seja na capacidade de campo $\left(\tau_{c c}\right)$, seja naquele em que a porosidade de aeração é igual a $10 \%\left(\tau_{\mathrm{Par}}\right)$; o limite superior é o menor valor de $\tau$ no qual a $\mathrm{RP}=2,0 \mathrm{MPa}\left(\tau_{\mathrm{RP}}\right)$ ou a $\tau$ correspondente ao ponto de murchamento permanente $\left(\tau_{\mathrm{PMP}}\right)$.

Os ajustes dos modelos das curvas de retenção deágua e de resistência do sol o à penetração foram feitos com base nos métodos de regressãolinear, por meio da rotina PROC REG (SAS, 1999). Com vistas em comparar o IOP e teor de CO do solo entre os tratamentos, utilizou-se o teste t para amostras independentes (Hatcher \& Stepanski, 1997).

\section{RESULTADOS E DISCUSSÃO}

Modificações nos teores de CO do solo estão associadas a diferentes fatores, em especial à intensidade de revolvimento do solo, podendo ter reflexos na forma e estabilidade da estrutura, com conseqüências no comportamento físico do solo. Os teores de CO do solo foram de $25,7 \mathrm{~g} \mathrm{~kg}^{-1}$ na SD e $21,4 \mathrm{~g} \mathrm{~kg}^{-1}$ na SDE, sendo estatisticamente 
diferentes $(p<0,05)$. Estes resultados indicam que a escarificação promoveu redução dos teores de CO do solo, em virtude do ambiente mais favorável à oxi dação do carbono (Bayer et al., 2003), concordando com os resultados encontrados por vários autores (Mahboubi et al., 1993; Beare et al., 1994).

Observou-se uma ampla faixa de valores de resistência do solo, necessária à model agem da curva de resistência do solo (Quadro 1). Os el evados valores do coeficiente de variação para resistência do solo à penetração justificam-se pel o gradiente de tensões de água, associados com a variação da densidade do solo, que permitem obter uma ampla faixa de valores de RP necessária à modelagem da curva deresistência do sol oà penetração. Resultados similares foram encontrados em outros estudos (Silva et al., 1994; Tormena et al., 1998, 1999a; I mhoff et al., 2001).

A densidade do solo apresentou distribuição normal peloteste deShapiro-Wilk para SD nas duas profundidades estudadas $(\mathrm{W}=0,9383, \mathrm{P}<\mathrm{W}=$ 0,$1128 ; W=0,9611, P<W=0,4027$ ) e para SDE na profundidade de 0,15-0,30 $\mathrm{m}(\mathrm{W}=0,9606, \mathrm{P}<\mathrm{W}=$ 0,3919). A exceção ocorreu na SDE na profundidade de 0-0,15 m, que apresentou comportamento próximo da normalidade $(\mathrm{W}=0,9145, \mathrm{P}<\mathrm{W}=$ $0,0275)$, o que pode ser atribuído ao efeito da escarificação no revolvimento do sol o nesta camada (Quadro 1).

$\mathrm{Na}$ profundidade de $0-0,15 \mathrm{~m}$, os valores de densidade do solo foram maiores no SD do que no SDE $(p<0,05)$ enquanto, na profundidade de $0,15-$ $0,30 \mathrm{~m}$, não foram constatadas diferenças entre os tratamentos. Resultados similares foram obtidos por Oliveira et al. (2001), Hamilton-Manns et al. (2002)
eT ormena et al. (2002). As diferenças de densidade do sol o na camada de 0-0,15 m indicam a persistência dos efeitos da escarificação, provavelmente associados à maior estabilidade estrutural do solo decorrente do maior crescimento radicular nesta camada. J á na camada de 0,15-0,30 m, a reconsolidação do solo, associada à menor eficiência da escarificação nesta camada, pode justificar a semelhança nos valores de densidade do solo. Segundo Evans et al. (1996), a densidade do solo, determinada pela técnica do anel volumétrico, éuma medida relativamente insensível dos efeitos da escarificação sobre o solo, o que pode justificar a ausência de diferenças entre os tratamentos na camada de 0,15-0,30 m.

No ajuste dos dados ao modelo de resistência (equações 3e4), constatou-se efeito estatisticamente significativo $(p<0,05)$ das variáveis qualitativas tratamento e profundidade nos coeficientes do modelo. O modelo matemático ajustado aos dados explicou 88 \% da variabilidade da resistência do solo à penetração, para ambos os tratamentos. Em consonância com Silva \& Kay (1997), foram obtidas as equações para cada tratamento e profundidade deamostragem (Quadro 2). Os val ores positivos dos coeficientes indicam que com o incremento da densidade do solo e da $\tau$ ocorreu aumento da resistência do solo à penetração, concordando com as constatações de vários pesquisadores (Boone et al., 1986, 1987; Boone, 1988; Boone \& Veen, 1994; Hakansson \& Lipiec, 2000; Lipiec \& Hakansson, 2000). Por meio destas funções, foi obtido para cada amostra, nos respectivos tratamentos e profundidades, o val or de $\tau$, no qual a $\mathrm{RP}=2,0 \mathrm{MPa}$, valor considerado impeditivo ao crescimento das raízes.

\section{Quadro 1. Densidade e resistência do solo à penetração na faixa de tensões matriciais utilizadas}

\begin{tabular}{|c|c|c|c|c|c|}
\hline Variável & Média & Desvio-padrão & Mínimo & Máximo & CV (\%) \\
\hline \multicolumn{6}{|c|}{ SD $0-0,15 \mathrm{~m}^{(1)}$} \\
\hline $\begin{array}{l}\mathrm{Ds}\left(\mathrm{Mg} \mathrm{m}^{-3}\right) \\
\operatorname{RP}(\mathrm{MPa})\end{array}$ & $\begin{array}{l}1,13 \\
2,28\end{array}$ & $\begin{array}{l}0,06 \\
1,76\end{array}$ & $\begin{array}{l}1,05 \\
0,50\end{array}$ & $\begin{array}{l}1,23 \\
8,23\end{array}$ & $\begin{array}{r}5,31 \\
77,19\end{array}$ \\
\hline \multicolumn{6}{|c|}{ SD $0,15-0,30 \mathrm{~m}^{(1)}$} \\
\hline $\begin{array}{l}\mathrm{Ds}\left(\mathrm{Mg} \mathrm{m}^{-3}\right) \\
\operatorname{RP}(\mathrm{MPa})\end{array}$ & $\begin{array}{l}1,14 \\
2,38\end{array}$ & $\begin{array}{l}0,05 \\
1,51\end{array}$ & $\begin{array}{l}1,05 \\
0,66\end{array}$ & $\begin{array}{l}1,24 \\
6,96\end{array}$ & $\begin{array}{r}4,39 \\
63,44\end{array}$ \\
\hline \multicolumn{6}{|c|}{ SDE $0-0,15 \mathrm{~m}^{(1)}$} \\
\hline $\begin{array}{l}\mathrm{Ds}\left(\mathrm{Mg} \mathrm{m}^{-3}\right) \\
\mathrm{RP}(\mathrm{MPa})\end{array}$ & $\begin{array}{l}1,09 \\
2,37\end{array}$ & $\begin{array}{l}0,05 \\
1,70\end{array}$ & $\begin{array}{l}0,95 \\
0,48\end{array}$ & $\begin{array}{l}1,17 \\
6,71\end{array}$ & $\begin{array}{r}4,59 \\
71,73\end{array}$ \\
\hline \multicolumn{6}{|c|}{ SDE $0,15-0,30 \mathrm{~m}^{(1)}$} \\
\hline $\begin{array}{l}\text { Ds }\left(\mathrm{Mg} \mathrm{m}^{-3}\right) \\
\operatorname{RP}(\mathrm{MPa})\end{array}$ & $\begin{array}{l}1,14 \\
2,83\end{array}$ & $\begin{array}{l}0,06 \\
1,78\end{array}$ & $\begin{array}{l}1,00 \\
0,64\end{array}$ & $\begin{array}{l}1,24 \\
7,19\end{array}$ & $\begin{array}{r}5,26 \\
62,90\end{array}$ \\
\hline
\end{tabular}

(1) Profundidade de amostragem do solo.

$\mathrm{N}$ =28; Ds = densidade do solo; RP = resistência do solo à penetração; CV = coeficiente de variação. SD = semeadura direta; SDE = escarificação do solo a $0,30 \mathrm{~m}$ de profundidade na semeadura direta. 
Quadro 2. Ajuste das equações da curva de resistência do solo à penetração e da curva de retenção de água, para os tratamentos semeadura direta (SD) e semeadura direta com escarificação (SDE ), nas profundidades de 0-0,15 m e 0,15-0,30 m, respectivamente

\section{Tratamento Profundidade Equação da curva de resistência do solo}

E quação da curva de retenção de água

$\mathrm{m}$

\begin{tabular}{|c|c|c|}
\hline SD & $0-0,15$ & $\mathrm{RP}=0,6867 \tau \tau^{0,2647} \mathrm{Ds} 8,8527$ \\
\hline SD & $0,15-0,30$ & $R P=1,2978 \tau \tau^{0,3148}$ Ds 4,1146 \\
\hline & $0-0,15$ & $\mathrm{RP}=1,3043 \tau \tau^{0,2647} \mathrm{Ds} 5,0463$ \\
\hline SD & $0,15-0,30$ & $\mathrm{RP}=2,4650 \tau 0,3148$ Ds 0,3082 \\
\hline
\end{tabular}

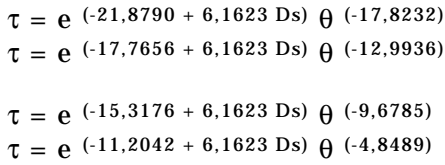

Para a curva de retenção de água no solo, o modelo ajustado aos dados explicou $74 \%$ da variabilidade da $\tau$ em relação à umi dade do sol o, para ambos os tratamentos. Observaram-se efeitos estatisticamente significativos dos tratamentos, da profundidade de amostragem e da densi dade do solo na curva de retenção. Constata-se que tanto o aumento da densidade do sol o quanto a diminuição da umidade resultam num aumento do valor da $\tau$ (Quadro 2), o qual, segundo Gupta et al. (1988), é devido à alteração na distribuição do tamanho de poros, com poros de menor diâmetro predominando com o aumento da densidade do solo. Além disso, os efeitos dos tratamentos e profundidade de amostragem, tanto na curva de retenção quanto na curva de resistência, indicam que a escarificação do solo alterou a distribuição dos poros do solo, com a magnitudedealteração dependendo da profundidade estudada.

As figuras 1 e 2 mostram as variações das tensões matriciais nos limites críticos correspondentes à capacidade de campo ( $\tau=0,01 \mathrm{MPa})$, à porosidade deaeração de $10 \%$, ao ponto de murcha permanente $(\tau=1,5 \mathrm{MPa})$ e à resistência do solo à penetração das raízes de 2,0 MPa, para cada val or de densidade do solo, nos tratamentos SD e SDE e nas profundidades de 0-0,15 m e 0,15-0,30 m.

Para a SD na profundidade de 0-0,15 m, o val or de $\tau$, no qual a porosidadeéde $0,10 \mathrm{~m}^{3} \mathrm{~m}^{-3}$, mantevese abaixo da tensão correspondente à capacidade de campo, até à densidade do solo de $1,20 \mathrm{Mg} \mathrm{m}^{-3}$ (Figura 1a). A partir deste valor de densidade do solo, é necessário que a $\tau$ atinja valores superiores aos correspondentes à capacidade de campo para manter um volume mínimo de poros com ar no solo, o que ocorreu em 14,3\% das amostras. I sto implica que a porosidade de aeração passa a determinar, a partir deste valor de densidade do solo, o limite inferior do IOP. Para o SDE (Figura lb), a $\tau$, na qual a porosidade é de $0,10 \mathrm{~m}^{3} \mathrm{~m}^{-3}$, não ultrapassou a $\tau$ correspondente à capacidade de campo, a qual determina o limite inferior do IOP. Resultados similares foram obtidos por Tormena et al. (1999b), que atribuíram este comportamento ao tipo e estabilidade da estrutura dos Latossolos, que preserva os espaços porosos necessários para que

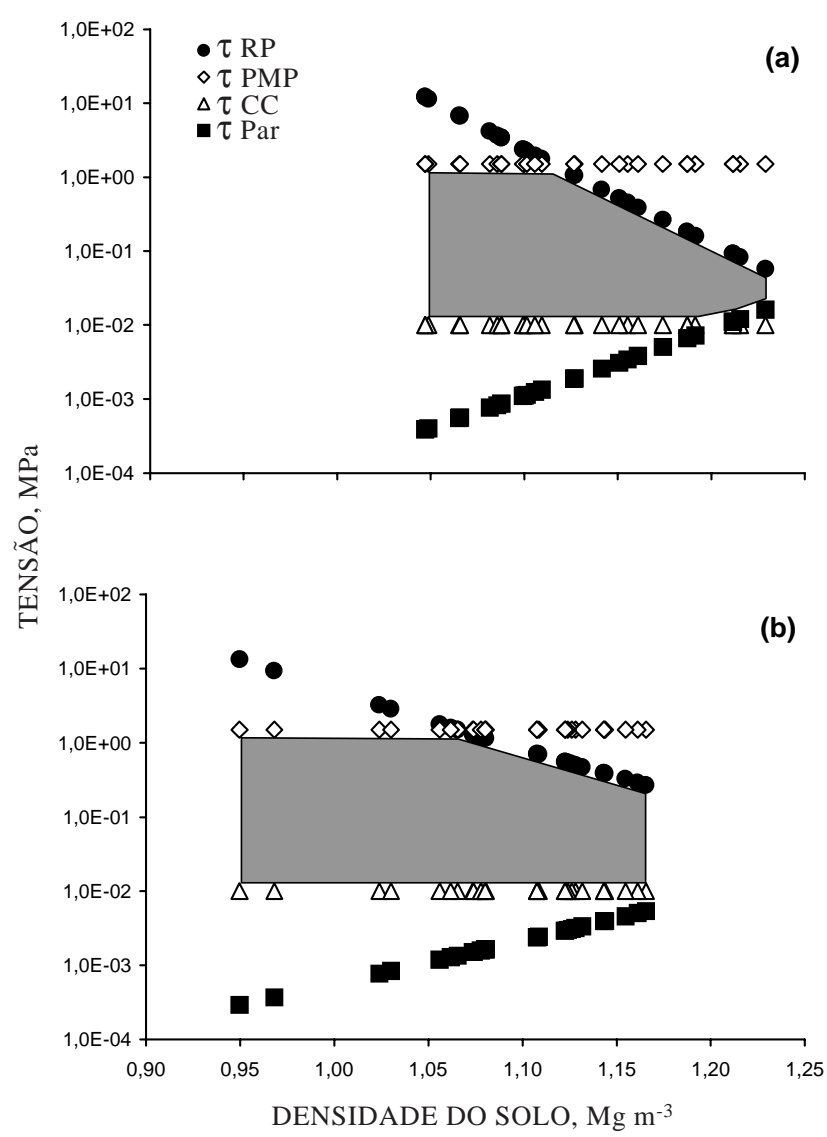

Figura 1. Variação do intervalo ótimo de tensão de água no solo (IOP) (área hachurada) no tratamento semeadura direta (SD) (a) e semeadura direta com escarificação (SDE) (b) na profundidade de $0-0,15 \mathrm{~m} . \tau_{\mathrm{RP}}=\tau$ em que $\mathrm{a}$ resistência à penetração $(R P)$ é igual a 2,0 $\mathrm{MPa}$; $\tau_{\mathrm{PMP}}=$ ponto de murcha permanente $(\tau=$ $1,5 \mathrm{MPa}) ; \tau_{\mathrm{cc}}=$ capacidade de campo $(\tau=$ $0,01 \mathrm{MPa}) ; \tau_{\mathrm{Par}}=\tau$ em que a porosidade de aeração é de $0,10 \mathrm{~m}^{3} \mathrm{~m}^{-3}$. 
ocorram as trocas gasosas no solo. I mhoff et al. (2001), analisando um solo arenoso, também constataram comportamento semel hante atribuindo-o à textura do solo.

O limite superior do IOP foi determinado pela $\tau$ correspondenteao ponto de murcha permanenteaté à densidade do solo de $1,11 \mathrm{Mg} \mathrm{m}^{-3}$ para o SD e $1,07 \mathrm{Mg} \mathrm{m}^{-3}$ para oSDE (Figura 1a,b). Para valores de densidade do solo superiores a estes, a resistência do solo à penetração substituiu a $\tau$ correspondente ao ponto de murcha permanente. Pode-se constatar ainda que a resistência do solo à penetração foi a variável física do solo que mais interferiu no IOP, pois se apresentou como limite superior deste em uma ampla faixa de variação da densidade do solo, atuando em 50 e 67,9 \% das amostras no SD eSDE, respectivamente (Figura 1 ). Este tipo de comportamento também foi observado por Tormena et al. (1998, 1999a,b), em solos com textura similar à do sol o utilizado nesteestudo, cultivado no sistema de semeadura direta, e também por Silva et al. (1994), Lipiec \& Hakansson (2000) e Imhoff et al. (2001), em sol os com diferentes texturas.

A pesar deterem apresentado a mesma tendência em relação à dependência da porosidade de aeração e da resistência do sol o à penetração de acordo com a densidade do solo, os tratamentos revelaram algumas diferenças que devem ser consideradas. Por exemplo, a $\tau$, em que a porosidade de aeração é de $10 \%$, não atuou comolimiteinferior doI OP noSDE, indicando o efeito da escarificação no aumento da macroporosidade do solo, como constatado por De Maria et al. (1999) e Watanabe et al. (2002). J á em relação à resistência do sol o à penetração, em ambos os tratamentos, esta se apresentou como o limite superior do IOP em mais de $50 \%$ das amostras. No tratamento SDE, a resistência do solo à penetração substituiu o ponto de murcha permanente em valores de densidade do sol o menores do que aqueles observados no tratamento SD. Estes resultados podem estar associados aos efeitos compressivos do escarificador, causando orientação das partículas de argila, o que magnifica o efeito da coesão com o secamento do solo. Os val ores médios do IOP foram de 0,92 $\mathrm{MPa}$, para o tratamento SD, e de 0,89 $\mathrm{MPa}$, para oSDE , não apresentando diferença estatística $(p>0,05)$.

Silva et al. (1994) indicaram que o valor de densidade do solo em que o IHO é igual a zero está associado a condições estruturais do sol o restritivas para o crescimento radicular, denominando-a densidade crítica do solo. Neste estudo, em ambos os tratamentos, não foram observados valores de densidade crítica do solo, indicando que, nos val ores de densidade do solo, tanto no SD quanto no SDE, as condições estruturais do solo são favoráveis ao crescimento das plantas e que, portanto, não há justificativas para a intervenção mecânica na SD por meio da escarificação do solo.
A variação das $\tau$ com a densidade do solo na SD e SDE, na profundidade de $0,15-0,30 \mathrm{~m}$, é descrita nas figura 2a e 2b, respectivamente. Na SD (Figura 2a), a $\tau$, na qual a porosidade de aeração é igual a $10 \%$, manteve-se abaixo da capacidade de campo até à densidade de $1,19 \mathrm{Mg} \mathrm{m}^{-3}$, ultrapassando a capacidade de campo em valores de densidade do solo superiores a esta. Nesta profundidade, isto ocorreu em $25 \%$ das amostras. Para a SDE (Figura 2b), verificou-se que, a partir de valores de densidade do solo superiores a $1,18 \mathrm{Mg} \mathrm{m}^{-3}$, a $\tau$ em que a porosidade de aeração é de $10 \%$, mostrou-se superior àquele correspondente à capacidade de campo e foi constatado em $21,4 \%$ das amostras, similar ao comportamento da SD (F igura 2a). Noque se refere à resistência do sol o à penetração, verificouse quena SD (F igura 2a), em val ores de densidade do solo acima de $1,08 \mathrm{Mg} \mathrm{m}^{-3}$, esta atingiu olimite crítico de 2,0 MPa, em $\tau$ inferiores àquela correspondente ao ponto de murcha permanente, estabelecendo o limite inferior do IOP em 85,7 \% das amostras.

Para o tratamento SDE (Figura 2b), os valores de $\tau$, em que a resistência do solo éigual a 2,0 MPa, foram inferiores ao ponto de murcha permanente, substituindo-o como limite superior em toda a extensão de valores de densidade do solo. Comportamento semel hante a este foi encontrado por Imhoff et al. (2001) em um solo de textura arenosa, cultivado com cana-de-açúcar, sendo tal comportamento atribuído ao sistema intensivo de cultivo do solo. Benjamin et al. (2003), em um solo de textura argilosa dos Estados Unidos, também relataram este tipo de comportamento. Tais resultados reafirmam a importância da resistência do solo à penetração em estudos da qualidade física do solo. Comparando as figuras 2a,b, constata-se maior amplitude do IOP na SD, com valores médios de 0,77 MPa na SD e de 0,44 MPa na SDE, os quais são estatisticamente diferentes $(p<0,05)$.

De acordo com a figura $2 a$, na SD, tanto a porosidade de aeração quanto a resistência do solo à penetração passaram a ser limitantes em valores de densidade do sol o próximos aos observados para a camada superficial (Figura 1a), demonstrando que, na SD a condição estrutural do solo éuniformeno perfil avaliado. Estes resultados corroboram os de Costa et al. (2003), que constataram não haver diferenças significativas na densidade e porosidade dosol o entre a camada superficial esubsuperficial de um Latossolo Bruno submetido ao sistema semeadura direta por 21 anos consecutivos. A partir daí, verifica-se que o manejo do solo no sistema semeadura direta por longos períodos detempo pode promover uniformidade no comportamento físico do solo nas camadas superficial esub-superficial equea escarificação, como no caso deste estudo, pode modificar negativamente a degradação da estrutura do solo, em sub-superfície.

De maneira geral, os resultados deste estudo indicam quea qualidade física do sol o foi homogênea 


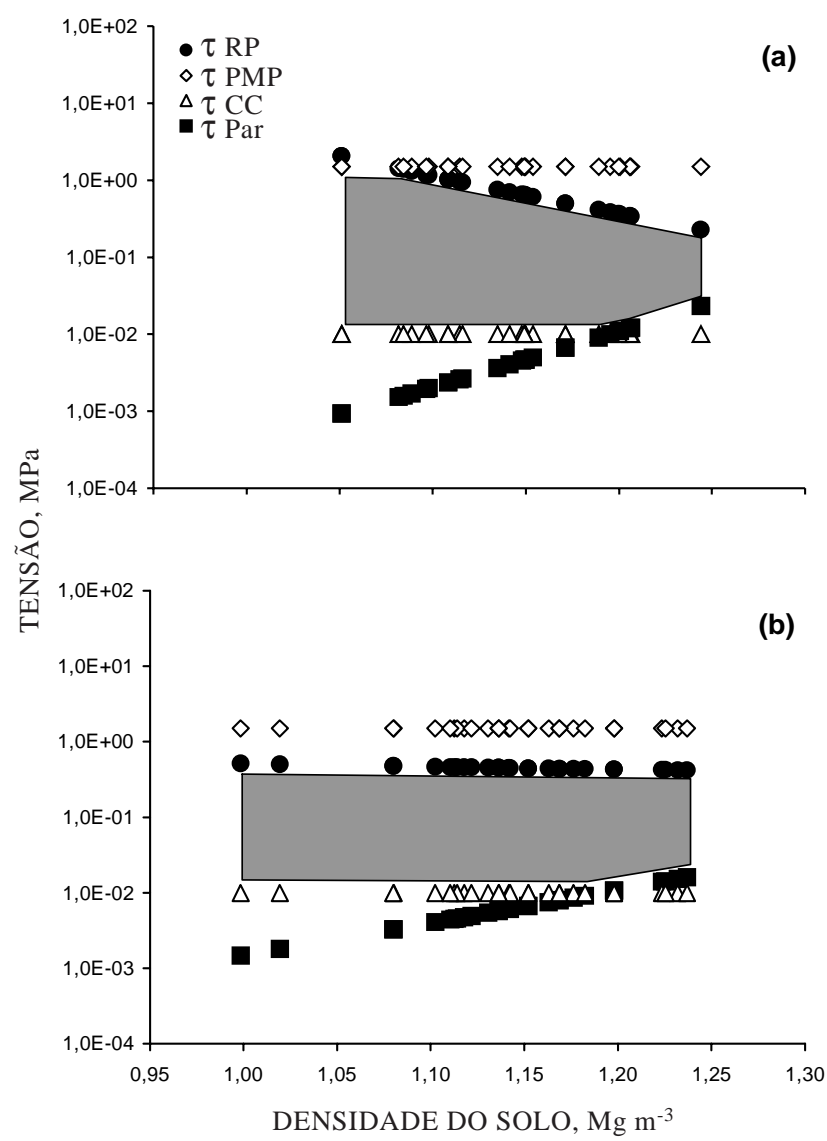

Figura 2. Variação do intervalo óti mo de tensão de água no solo (IOP) (área hachurada) no tratamento semeadura direta (SD) (a) e semeadura direta com escarificação (SDE) (b) na profundidade de $0,15-0,30 \mathrm{~m} . \tau_{\mathrm{RP}}=\tau$ em que a resistência à penetração $(R P)$ é igual a 2,0 MPa; $\tau_{\mathrm{PMP}}=$ ponto de murcha permanente $(\tau=1,5 \mathrm{MPa}) ; \tau_{\mathrm{cC}}=$ capacidade de campo $(\tau=$ $0,01 \mathrm{MPa}) ; \tau_{\mathrm{Par}}=\tau$ em que a porosidade de aeração é de $0,10 \mathrm{~m}^{3} \mathrm{~m}^{-3}$.

no perfil avaliado do tratamento SD comparado com a SDE, e isto se deve provavel mente à ação efetiva da reconsolidação do solo no tratamento SDE e ao maior teor de CO do solo encontrado na SD. No entanto, éimportantesalientar que, em nenhum dos tratamentos, em nenhuma das profundidades estudadas, o IOP apresentou valores nulos, ou seja, mesmo com as diferenças entre os tratamentos, não foram encontrados valores de densi dade do sol o em que o IOP $=0$, discordando dos resultados obtidos por Tormena et al. (1998, 1999a,b), Imhoff et al. (2001) e Benjamin et al. (2003). Todavia, apesar da ausência de valores de densidade do solo crítica, é considerável o fato de que a escarificação promoveu efeitos deletérios na qualidade estrutural do solo, relacionados principal mentecom a resistência do solo à penetração com a maior intensidade na camada sub-superficial.

\section{CONCLUSÕES}

1. A escarificação do solo promoveu efeitos benéficos na camada de 0-0,15 m principalmente em relação à porosidade de aeração. No entanto, na camada de 0,15-0,30 m, a escarificação reduziu o intervalo ótimo de tensão de água no solo (IOP), principalmente em virtude da resistência do solo à penetração que se comportou como limite superior em toda a faixa de variação do IOP.

2. Os resultados sugerem que, neste solo e sob esta condição de manejo, não é necessária a escarificação.

\section{LITE RATURA CITADA}

BAYER, C.; MARTIN-NETO, L. \& SAAB, S.C. Diminuição da humificação da matéria orgânica de um Cambissolo Húmico em plantio direto. R. Bras. Ci. Solo, 27:537-544, 2003.

BEARE, M.H.; HENDRIX, P.F \& \& COLEMAN, D.C. Water-stable aggregates and organic matter fractions in conventional and no-tillage soils. Soil Sci. Soc. Am. J ., 58:777-786, 1994.

BENJ AMIN, J .G.; NIELSON, D.C. \& VIGIL, M.F. Quantifying effects of soil conditions on plant growth and crop production. Geoderma, 116:137-148, 2003.

BEUTLER, A.N.; SILVA, M.L.N.; CURI, N.; FERREIRA, M.M.; CRUZ, J.C. \& PEREIRA FILHO, I.A. Resistência à penetração e permeabilidade de Latossolo Vermelho distrófico típico sob sistemas de manejo na região dos cerrados. R. Bras. Ci. Solo, 25:167-177, 2001.

BLAKE, G.R.; HARTGE, K.H. Bulk density. In: KLUTE, A., ed. Methods of soil analysis: physical and mineralogical methods. 2.ed. Madison: America Society of Agronomy, 1986. p.363-375.

BOONE, F.R. Wheater and other enviromental factors influencing crop responses to tillage and traffic. Soil Till. Res., 11:283-324, 1988.

BOONE, F.R.; van der WERF, H.M.G.; KROESBERGEN, B.; TEN HAAG, B.A. \& BOERS, A. The effect of compaction of arable layer in sandy soils on the growth of maize for silage. I. Critical potentials in relation to soil aeration and mechanical impedance. Neth. J. Agric. Res., 34:155-171, 1986.

BOONE, F.R.; van der WERF, H.M.G.; KROESBERGEN, B.; TEN HAAG, B.A. \& BOERS, A. The effect of compaction of the arable layer in sandy soils on the growth of maize for silage. II. Soil conditions and plant growth. Neth. J . Agric. Res., 35:113-128, 1987.

BOONE, F.R. \& VEEN, B.W. Mechanisms of crop responses to soil compaction. In: SOANE, B.D. \& VAN OUWERKERK, C., eds. Soil compaction in crop production. Amsterdam, Elsevier, 1994. cap.11, p.237-264.

BUSSCHER, W.J . Adjustment of flat-tipped penetrometer resistance data to common water content. Trans. Am. Soc. Agric. Eng., 3:519-524, 1990. 
BUSSCHER, W.J .; BAUER, P.J . \& FREDERICK, J.R. Recompaction of a coastal loamy sand after deep tillage as a function of subsequent cumulativerainfall. Soil Till. Res., 68:49-57, 2002.

BUSSCHER, W.J .; EDWARDS, J .H.; VEPRASKAS, M.J . \& KARLEN, D.L. Residual effects of slit tillageand subsoiling in a hardpan soil. Soil Till. Res., 35:115-123, 1995.

COSTA, F.S.; ALBUQUERQUE, J .A.; BAYER, C.; FONTOURA, S.M.V. \& WOBETO, C. Propriedades físicas de um Latossolo Bruno afetadas pelos sistemas plantio direto e preparo convencional. R. Bras. Ci. Solo, 27:527-535, 2003.

DE MARIA, I.C.; CASTRO, O.M. \& SOUZA DIAS, H. Atributos físicos do solo e crescimento radicular de soja em Latossolo Roxo sob diferentes métodos de preparo do solo. R. Bras. Ci. Solo, 23:703-709, 1999.

EMPRESA BRASILEIRA DE PESQUISA AGROPECUÁRIA EMBRAPA. Manual de métodos de análises de solos. 2.ed. Rio de J aneiro, 1997. 212p.

EMPRESA BRASILEIRA DE PESQUISA AGROPECUÁRIA E mbrapa. Sistema Brasileiro de Classificação de Solos. Rio de J aneiro, Ministério da Agricultura e do Abastecimento, 1999. 412p.

EVANS, S.D.; LINDSTROM, M.J .; VOORHEES, W.B.; MOCRIEF, J .F. \& NELSON, G.A. Effect of subsoiling and subsequent tillage on soil bulk density, soil moisture and corn yield. Soil Till. Res., 38:35-46, 1996.

FEDERAÇÃO BRASILEIRA DE PLANTIO DIRETO NA PALHA - FEBRAPDP. Disponível em <http:// www.febrapdp.org.br>. Acesso em: 08 mar. 2004.

GRABLE, A.R. \& SIEMER, E.G. Effects of bulk density, aggregate size, and soil water suction on oxygen diffusion, redox potential and elongation of corn roots. Soil Sci. Soc. Am. J ., 32:180-186, 1968.

GRANATSTEIN, D. \& BEZDICEK, D.F. The need for a soil quality index: local and regional perspectives. Am. J . Altern. Agric., 7:12-16, 1992.

GUPTA, S.C.; SHARMA, P.P. \& FRANCHI, S.A. Compaction effects on soil structure. Adv. Agron., 42:331-338, 1988.

HAKANSSON, I. \& LIPIEC, J. A review of the usefulness of relative bulk density values in studies of soil structure and compaction. Soil Till. Res., 53:71-85, 2000.

HAMILTON-MANNS, M.; ROSS, C.W.; HORNE, D.J . \& BAKER, C.J. Subsoil loosening does litle to enhance the transition to no-tillage on a structurally degrade soil. Soil Till. Res., 68:109-119, 2002.

HATCHER, L. \& STEPANSKI, E.J . A step-by-step approach to using the SAS System for Univariate and Multivariate Statistics. Cary, SAS Institute, 1997. 552p.

HILL, R.L. Long-term conventional and no-tillage effects on selected soil physical properties. Soil Sci. Soc. Am. J., 54:161-166, 1990.

IMHOFF, S.; DA SILVA, A.P.; DIAS JÚNIOR, M.S. \& TORMENA, C.A. Quantificação de pressões críticas para o crescimento de plantas. R. Bras. Ci. Solo, 25:11-18, 2001.
J ENSEN, C.R.; MOGENSEN, V.O.; POULSEN,H.H.; HENSEN, I.E.; AAGOT, S.; HANSEN, E.; ALI, M. \& WOLLENWEBER, B. Soil water matric potencial rather than water content determines drought responses in fieldgrown lupin (Lupinus angustifolius). Aust. J . Plant Phys., 25:353-363, 1998.

KARLEN, D.L. \& STOTT, D.E. A framework for avaluating physical and chemical indicators of soil quality. In: DORAN, J. W., COLEMAN, D. C., BEZDICEK, D. F. \& STEWART, B. A., eds. Defining soil quality for a sustainable environment. Madison, Soil Science Society of America, 1994. p.53-72. (SSSA Special Publication, 35)

KAY, B.D. Rates of changes of soil structure under different cropping systems. Adv. Soil Sci., 12:1-51, 1990.

KAY, B.D. \& ANGERS, D.A. Soil structure. In: A. SUMNER, M.E., ed. Handbook of Soil Science. Boca Raton, CRC Press, 1999. p.229-276.

KIEHL, E.J . Manual de edafologia - relações solo-planta. São Paulo, Editora Agronômica Ceres, 1979. 262p.

KIRKEGAARD, J.A.; MUNNS, R.; J AMES, R.A.; GARDNER, P.A. \& ANGUS, J .F. Reduced growth and yield of wheat with conservation cropping II: Soil biological factors limit growth under direct drilling. Aust. J . Agric. Res. 46:75-88, 1995.

KLUTE, A. Water retention: Laboratory Methods. In: KLUTE, A., ed. Methods of soil analysis - physical and mineralogical methods. Madison, America Society of Agronomy, 1986. p.635-660.

LETEY, J . Relationship between soil physical properties and crop production. Adv. Soil Sci., 1:277-294, 1985.

LIPIEC, J. \& HAKANSSON, I. Influences of degree of compactness and matric water tension on some important plant growth factors. Soil Till. Res., 53:87-94, 2000.

MAHBOUBI, A.A. \& LAL, R. Long-term tillage effects on changes in structural properties of two soils in central Ohio. Soil Till. Res., 45:107-118, 1998.

MAHBOUBI, A.A.; LAL, R. \& FAUSSEY, N.R. Twenty-eigth years of tillage effects on two soil in Ohio. Soil Sci. Soc. Am. J ., 57:506-512, 1993.

MÜLLER, M.M.L.; CECCON, G. \& ROSOLEM, C.A. Influência da compactação do solo em subsuperfície sobre o crescimento aéreo e radicular de plantas de adubação verde de inverno. R. Bras. Ci. Solo, 25:531-538, 2001.

OLIVEIRA, J .O.A.P.; VIDIGAL FILHO, P.S.; TORMENA, C.A.; PEQUENO, M.G.; SCAPIM, C.A.; MUNIZ, A.S. \& SAGRILO, E. Influência de sistemas de preparo do sol o na produtividade da cultura da mandioca (Manihot esculenta, CRANTZ). R. Bras. Ci. Solo, 25:443-450, 2001.

REICHARDT, K. Capacidade decampo. R. Bras. Ci. Solo, 12:211216, 1988.

ROSS, P.J .; WILLIANS, J . \& BRISTOW, K.L. Equations for extending water-retention curves to drynees. Soil Sci. Soc. Am. J ., 55:923-927, 1991.

SAS INSTITUTE. SAS/STAT procedure guide for personal computers. 5.ed. Cary, 1999. 
SAVAGE, M.J .; RITCHIE, J .T.; BLAND, W.L. \& DUGAS, W.A. Lower limit of soil water availability. Agron. J ., 88:844851, 1996.

SHARMA, P.K. \& BHUSHAN, L. Physical characterization of a soil amended with organic residues in a rice-wheat cropping system using a single value soil physical index. Soil Till. Res., 60:143-152, 2001.

SILVA, A.P. \& KAY, B.D. Estimating the least limiting water range of soils from properties and management. Soil Sci. Soc. Am. J ., 61:877-883, 1997.

SILVA, A.P.; KAY, B.D. \& PERFECT, E. Characterization of theleast limiting water range. Soil Sci. Soc. Am. J ., 58:17751781, 1994

STONE, L.F. \& SILVEIRA, P.M. Efeitos do sistema de preparo e da rotação de culturas na porosidade e densidade do solo. R. Bras. Ci. Solo, 25:395-401, 2001.

TAYLOR, H.M.; ROBERSON, G.M. \& PARKER J r, J J . Soil strength-root penetration relations to medium to coarsetextured soil materials. Soil Sci., 102:18-22, 1966.

TORMENA, C.A.; SILVA, A.P.; GONÇALVES, A.C.A. \& FOLEGATTI, M.V. Intervalo ótimo de potencial da água do solo: um conceito para avaliação da qualidade física do solo e manejo da água na agricultura irrigada. R. Bras. Eng. Agríc. Ambiental, 20:286-292, 1999a.

TORMENA, C.A.; SILVA, A.P. \& LIBARDI, P.L. Caracterização do interval o hídrico ótimo de um Latossolo Roxo sob plantio direto. R. Bras. Ci. Solo, 22:573-581, 1998.

TORMENA, C.A.; SILVA, A.P. \& LIBARDI, P.L. Soil physical quality of a Brazilian Oxisol under two tillagesystems using the least limiting water range approach. Soil Till. Res., 52:223-232, 1999b.
TORMENA, C.A.; BARBOSA, M.C.; COSTA, A.C.S. \& GONÇALVES, A.C.A. Densidade, porosidade resistência à penetração em Latossolo Vermelho distrófico sob diferentes sistemas de preparo do solo. Sci. Agric., 59:795801, 2002.

TORRES, E. \& SARAIVA, O.F. Estudo das causas da compactação do solo e do seu efeito sobre a soja. In: EMPRESA BRASILEIRA DE PESQUISA AGROPECUÁRIA. Resultados de pesquisa da Embrapa Soja. EMPRESA BRASILEIRA DE PESQUISA AGROPECUÁRIA, Londrina, 1998. p.177-181.

TORRES, E.; SARAIVA, O.F.; PICCININ, J .L.; FARIAS, J .R.B.; GALERANI, P.R. \& GAZZIERO, D.L.P. Avaliação de sistemas de preparo do solo, rotação de culturas e semeadura da soja. In: Resultados de pesquisa da E mbrapa Soja. EMPRESA BRASILEIRA DE PESQUISA AGROPECUÁRIA, Londrina, 1998. p.171-177.

TWONLOW, S.J .; PARKINSON, R.J . \& REID, I. Temporal changes in soil physical conditions after deep loosening of a silty clay loam in SW England. Soil Till. Res., 31:31-47, 1994.

WATANABE, S.H.; TORMENA, C.A.; ARAUJ O, M.A.; VIDIGAL FILHO, P.S.; PINTRO, J.C.; COSTA, A.C.S. \& MUNIZ, A.S. Propriedades físicas de um Latossolo Vermelho distrófico influenciadas por sistemas de preparo do solo utilizados para a implantação da cultura da mandioca. Acta Sci., 24:1255-1264, 2002.

WU, L.; FENG, G.; LETEY, J .; FERGUSON, L.; MITCHELL, J .; MCCULLOUGH-SANDEN, B. \& MARKEGARD, G. Soil management effects on the nonlimiting water range. Geoderma, 114:401-414, 2003. 